\title{
Uniqueness of Inverse Scattering Problem in Local Quantum Physics
}

\author{
dedicated to Moyses Nussenzveig on the occasion of his 70th birthday \\ Bert Schroer \\ present address: CBPF, Rua Dr. Xavier Sigaud 150, \\ 22290-180 Rio de Janeiro, Brazil \\ emailschroer@cbpf.br \\ permanent address: Institut für Theoretische Physik \\ FU-Berlin, Arnimallee 14, 14195 Berlin, Germany
}

February 2003

\begin{abstract}
It is shown that the operator algebraic setting of local quantum physics leads to a uniqueness proof for the inverse scattering problem. The important mathematical tool is the thermal KMS aspect of wedge-localized operator algebras and its strengthening by the requirement of crossing symmetry for generalized formfactors. The theorem extends properties which were previously seen in $d=1+1$ factorizing models.
\end{abstract}

\section{Inverse Problem in LQP}

Most inverse problems have their origin in classical physics where they result from the question to what extend scattering (asymptotic) data allow a reconstruction of local data. The universality and importance of this kind of quest inspired Marc Kac to the famous aphorism "How to hear the shape of a drum" which refers to Weyl's problem associated with geometric reconstructions from the asymptotic distribution of eigenvalues of the Laplace-Beltrami operator. In its present use it incorporates a wide range of problems concerning the partial or complete determination of local data from a seemingly weaker asymptotic input. In local quantum physics also the problem of how to re-construct the full content of QFT (in particular the superselection aspects of charge-carrying fields) from its "observable shadow" i.e. the so-called DR-theory [1] may be viewed as an inverse problem.

The inverse problem in the present context is the question to what extends the data contained in a physically admissible (unitary, crossing symmetry,...) S-matrix determines a quantum field theory. Since it is easy to see that an 
S-matrix cannot be uniquely related to one field but rather is shared by very big equivalence classes of local fields, it is clear that the first step in such an investigation is to formulate QFT in a way that two isomorphic theories whose different appearance is only due to the use of different "field-coordinatizations" are easily recognizable as being one and the same. This is not possible (or rather extremely cumbersome) in the standard approach based on pointlike fields.

Fortunately there exists such a framework which pays due attention to fields belonging to the same local equivalence class (fields from the same Borchers class [2] [3]) and which therefore generate the same system of local algebras as the given field. It is known under two different names algebraic quantum field theory (AQFT) or local quantum physics (LQP) 3]; in fact one of the motivations behind its creation was precisely a better understanding of the insensitivity of the S-matrix against certain kind of local changes of field-coordinatizations. Its relation to standard QFT is similar to that of coordinate-based differential geometry to its more modern coordinate-free intrinsic formulation. Here as there one retains all the underlying principles and only introduces additional more elegant concepts to implement them. If one wants to emphasize that one deals with QFT but without the reader immediately thinking in terms of Lagrangians and functional integral representations it is quite helpful to use AQFT or LQP instead of QFT (a name as "intrinsic QFT" or "field-coordinatization-free QFT" would appear a bit clumsy).

Using the powerful mathematical tool of Tomita-Takesaki theory adapted to wedge algebras 12 as well as the closely related crossing symmetry, we show that if a solution exists at all, it is necessarily unique.

This result generalizes previous special findings in $d=1+1$ models (for a review in the LSZ setting of scattering theory see 31]) concerning the modular derivation of the wedge localization interpretation [4] [5] of the ZamolodchikovFaddeev algebra [6] in $\mathrm{d}=1+1$ in which case one also is able to control a good part of the explicit construction of the associated QFT. For such models Zamolodchikov and Faddeev observed that the structure of their factorizing S-matrix can be encoded into an interesting algebraic structure. Although the objects which generate this algebra were left without a physical interpretation, they seemed to constitute a quite useful technical tool for the construction of generalized formfactors (matrix elements of operators between physical multi-particle state vectors) affiliated with the model S-matrix [7. The application of modular theory to this situation revealed that the rapidity dependent Z-F operators are special cases of "(vacuum) polarization-free-generators" (PFG) for the wedgelocalized algebra [4] [5]. In this special setting of "tempered PFGs", the relation between the crossing symmetry in momentum space as formulated in the LSZ theory and the KMS thermal condition following from Bisognano-Wichmann properties of wedge algebras is well-understood ${ }^{1}$.

In the general case the PFG's have unwieldy domain properties [9] which prevent their use in relating momentum space crossing with the spacetime KMS

\footnotetext{
${ }^{1}$ For the case of absense of bound states, a detailed and mathematically rigorous use of the modular theory (which takes into account the domain problems of unbounded operators affiliated with $\mathcal{A}(W)$ ) can be found in a recent paper [8].
} 
property of wedge localization. In this case we will separately assume the validity of the crossing property and show that if we combine this with the deduced thermal KMS for wedge algebras we may obtain the desired uniqueness of the LQP (without being presently able to say something about the existence). It should not be a surprise that with our assumptions being stronger than those used in 11] we also get a stronger statement. For example we exclude in case of $\mathrm{S}=1$ any would be solution beyond Wick-polynomials or in a more algebraic language beyond the free Borchers-class.

\section{The use of modular theory in wedge localiza- tion}

The new tool on which the proof relies in an essential way is the modular theory of operator algebras as discovered and elaborated by Tomita and Takesaki ${ }^{2}$.

Since even in the setting of QFT the word "modular" occurs with different meanings, we will briefly define its present use. Actually the more common use is that of "modular invariance" in the more special setting of chiral conformal field theory. Although this is not its present meaning, a future deep connection of the T-T operator modular theory to this causality-related classification tool of "modular invariance" for chiral conformal models [13] is by no means excluded.

Let us start from a special geometric situation encountered in QFT. Consider the $x_{0}-x_{1}$ wedge $W_{0}$ in d-dimensional Minkowski spacetime which is defined by taking convex combination of the two light rays generators $( \pm 1,1,0 . .0)$ and on which the affiliated Lorentz boost $\Lambda_{W_{0}}(\chi)$ acts as a wedge-preserving diffeomorphism. Imagine that a quantum field theory is given to us and we are interested to study the structure of the $\mathcal{A}\left(W_{0}\right)$ subalgebra which the fields generate if we limit the support of smearing functions to the wedge region (the rigorous aspects of operator algebras will be postponed to later). The boost acts on this wedge algebra as a unitary implemented automorphism. Let us change the rapidity parametrization by the factor $2 \pi$ and write the unitary representation in QFT in terms of a selfadjoint $K$ boost generator as

$$
\Delta^{i t} \equiv U\left(\Lambda_{W_{0}}(\chi=2 \pi t)\right)=e^{2 \pi i t K}
$$

We then may introduce an unbounded positive operator $\Delta^{\frac{1}{2}}$ by "analytic continuation in t", which in functional calculus terms means that we are restricting the Hilbert space to those vectors $\psi$ which upon action with $\Delta^{i t}$ lead to a vectorvalued function $\psi(t) \equiv \Delta^{i t} \psi$ which is continuous in $-i \pi \leq \operatorname{Im} t \equiv \theta \leq 0$ and analytic on the open strip $-i \pi<\theta<0^{3}$. In addition to the wedge related boost, we also consider the antiunitary (since it involves time reversal) reflection along

\footnotetext{
${ }^{2}$ For an eyewitness account of the birth of this theory as well as a mathematical physics presentation of its content see 12 .

${ }^{3}$ Most of the analyticity properties in $\mathrm{x}$ - or momentum- space rapidities result from domain properties of unbounded operators.
} 
the edge

$$
\begin{aligned}
& J \equiv U(r) \\
& r: x_{0}, x_{1} \rightarrow-x_{0},-x_{1}
\end{aligned}
$$

which up to a $\pi$-rotation around the $e_{1}$-axis is identical to the famous TCP transformation of particle physics. Since this transformation commutes with the boost $\Delta^{i t}$, its antiunitarity leads to the commutation relation (always on the relevant domains)

$$
\begin{gathered}
J K=-K J \\
J \Delta^{\frac{1}{2}}=\Delta^{-\frac{1}{2}} J
\end{gathered}
$$

on the respective domains of definition. This in turn yields

$$
\begin{gathered}
S^{2} \subset 1 \\
\text { for } S \equiv J \Delta^{\frac{1}{2}}
\end{gathered}
$$

i.e. we encounter the rare case (not even to be found in extensive textbooks on mathematical physics as that by Reed-Simon) of an unbounded antilinear operator which is involutive and "transparent" on its domain i.e. $\operatorname{Dom}(S)=$ Range $(S)$. In a moment we will see that it is just this somewhat exotic property which enables the encoding of concrete spacetime geometric information concerning quantum localization into abstract domain properties.

It is the content of a theorem (the Bisognano-Wichmann theorem [3]) that this operator is Tomita's famous S-involution for the operator algebra which the local fields generate if one restricts the smearing functions to have support in the wedge. Namely in terms of the affiliated von Neumann algebra $\mathcal{A}\left(W_{0}\right)$ the $S$ fulfills the defining relation 3 [12]

$$
S A \Omega=A^{*} \Omega
$$

where $\Omega$ is the vacuum vector, the star denotes the standard Hermitian adjoint in operator algebras and $\mathrm{S}$ turns out to be closable (where the same letter is alsso used for its closure) whose polar decomposition leads precisely to modular objects $\Delta^{\frac{1}{2}}, J$ which are related to the unitary boost operator and the TCP related antiunitary implementer of the reflection along the edge of the wedge. This is a special case of the Tomita Takesaki modular theory whose prerequisite is the existence of a von Neumann algebra in "general position" i.e. a pair $(\mathcal{A}, \Omega)$ with $\Omega$ being a cyclic and separating vector for $\mathcal{A}^{4}$. From this input Tomita and Takesaki derive:

\footnotetext{
${ }^{4}$ Physicists who independently developed these concepts, often (especially in chiral conformal field theory) talk about the unique "operator-statevector relation" $A \longleftrightarrow A \Omega$; the correct mathematical backing is the reference to the Reeh-Schlieder theorem [2. It is often overlooked that the relation is not universal but depends on the chosen local algebras $\mathcal{A}(\mathcal{O})$.
} 
- The Tomita operator $S$ defined by (5) is a densely defined closed antilinear involution whose polar decomposition $S=J \Delta^{\frac{1}{2}}$ leads to an antiunitary reflection $J$ (abstract generalization of a TCP reflection) and modular dynamics $\Delta^{i t}$ (abstract generalization of a Hamiltonian). In contrast to our geometric approach which started from properties of boosts and reflections along the edge of a wedge. The Tomita setting is abstract in that $S$ is not defined geometrically but rather by association with a "standard pair" $(\mathcal{A}, \Omega)$ in operator algebra theory. The standardness of the wedge algebra together with the vacuum state in QFT is nothing else than a special realization of the famous Reeh-Schlieder property in local QFT.

- The $J, \Delta^{i t}$ have the following significance with respect to the operator algebra

$$
\begin{aligned}
A d J \mathcal{A} & =\mathcal{A}^{\prime} \\
A d \mathcal{A} & =\sigma_{t}(\mathcal{A})
\end{aligned}
$$

Here as in the sequel the upper dash on an operator algebra denotes its commutant, the adjoint action of the modular unitary $\Delta^{i t}$ implements the modular group $\sigma_{t}(\cdot) \equiv A d \Delta^{i t}$ which only depends on the state $\omega(A)=$ $(\Omega, A \Omega), A \in \mathcal{A}$ and not its implementing vector $\Omega$.

- A necessary and sufficient condition for the standardness (cyclicity+separating property) of the pair $(\mathcal{A}, \Omega)$ is the thermal KMS property in terms of the state $\omega$ is: there exists a $2 \pi$-open-strip analytic function (continuous in the closed strip ) $F_{A, B}(z)$ with

$$
\begin{aligned}
F_{A, B}(t) & \equiv \omega\left(\sigma_{t}(A) B\right) \\
\omega\left(B \sigma_{t}(A)\right) & =\lim _{z \rightarrow t+i} F_{A, B}(z)
\end{aligned}
$$

The above theorem of Bisognano and Wichmann may now be rephrased as saying that those operator wedge algebras which are generated by covariant fields do have a geometric modular theory.

In more recent times there have been successful attempts to establish these geometric modular aspects of wedge algebras directly in the seemingly more general setting of algebraic QFT which avoids the use of fields already at the start 26]. The validity of this KMS condition (with the modular group acting geometrically as the Lorentz-boost) is sufficient for establishing that also $J$ acts geometrically i.e. that the von Neumann commutant is localized in the geometrically opposite wedge $W^{\prime}$ (Haag duality) $\mathcal{A}(W)=\mathcal{A}\left(W^{\prime}\right)^{\prime}$.

Starting with one standard wedge algebra $\left(\mathcal{A}\left(W_{0}\right), \Omega\right)$, the Poincaré group generates a net of wedge algebras $(\mathcal{A}(W), \Omega)_{W \subset \mathcal{W}}$ and vice versa, a net of wedge algebras whose modular data fulfill the prerequisites of the $\mathrm{B}-\mathrm{W}$ theorem, generate a Poincaré group symmetry which is uniquely determined from the modular groups of the wedges [12. In order to extract sufficient physical informations one needs nets for smaller compact causally closed regions. A net 
of double cones $D$ may be defined in terms of intersections

$$
\mathcal{A}(D) \equiv \cap_{D \subset W} \mathcal{A}(W)
$$

In order to achieve our goal we must be able to relate the wedge algebra with the scattering operator $S_{s c}$. This is possible in the LSZ framework of QFT because although the representation theory of the connected Poincaré-group for the incoming (outgoing) free fields is the same as for the interacting Heisenberg fields, this is not so for the reflections involving time reversal. In particular the $J$ in (3) which represents the wedge reflection in the presence of interactions is different from its interaction-free asymptotic counterpart [4] $J_{\text {in }}$

$$
J=S_{s c} J_{i n}
$$

This implies that in the characterization of the wedge-localized (dense) subspace:

$$
\begin{aligned}
H(W) & =H_{R}(W)+i H_{R}(W) \\
H_{R}(W) & =\text { real subspace }\{\psi \mid S \psi=\psi\} \\
S\left(\psi_{1}+i \psi_{2}\right) & =\psi_{1}-i \psi_{2}, S=S_{s c} S_{i n}
\end{aligned}
$$

the position of the dense subspace $H(W)$ inside the total Hilbert space depends in a subtle way on the interaction through $S_{s c}$. The domain of $\Delta^{\frac{1}{2}}$ is now encoded more concretely in terms of the complex dense space $H(W)$ whose real and imaginary part are vectors in a closed real subspace $H_{R}(W)$. These real closed subspaces encode the full spatial aspect of wedge localization. With the help of the graph of the Tomita involution $S$ one may even introduce a topology in terms of which the dense subspace becomes a Hilbert space in its own right ${ }^{5}$, but all these spatial concepts are still quite remote from the task of characterizing a wedge algebra uniquely in terms of the scattering matrix. The reason is the following. The algebra-state vector relation $A \longleftrightarrow A \Omega$ is not universal but changes with the algebra (even within the family of wedges). In particular the spatial modular theory without additional informations is too weak to uniquely determine an operator algebra. However the use of scattering theory is sufficient for obtaining a pure algebraic derivation of the BisognanoWichmann geometric properties for the modular objects without reference to pointlike field coordinatizations 27.

Connes has given a criterion [28] which allows to obtain from the spatial modular theory an algebra with the same modular objects. This is achieved by controlling certain facial properties of subcones of a natural cone $\mathcal{P}(\mathcal{A}(W))$ associated with $H_{R}(W)$. But one presently lacks a physical foundation and justification for such a procedure. Fortunately for our interest in uniqueness, these difficulties can be avoided if one assumes an additional physically motivated working hypothesis. This is the crossing property of particle matrix elements (formfactors) of wedge localized operators. For this we need to remind the reader of a bit of scattering theory adapted to the algebraic framework.

\footnotetext{
${ }^{5} H(W)$ with the $S$-graph norm may be called the thermal Hilbert space, because it offers a natural description of the (Hawking-Unruh) thermal aspects of the vacuum upon its restriction to the wedge algebra.
} 


\section{Uniqueness from KMS-thermality and cross- ing}

It is well-known [9] that any vector $\psi$ which is in the domain of the positive "analytically continued" standard L-boost (11) $\Delta_{W}^{\frac{1}{2}}$ (which is defined by the use of the functional calculus of spectral theory on the selfadjoint boost generator) has a unique relation to an (generally unbounded) operator $F_{\psi, \mathcal{A}(W)}$ affiliated with $\mathcal{A}(W)$ with

$$
F_{\psi, \mathcal{A}(W)} \Omega=\Psi, F_{\psi, \mathcal{A}(W)}^{*} \Omega=S_{W} \Psi
$$

But this famous statevector-operator relation depends crucially on the standard pair $(\mathcal{A}(W), \Omega)$. If the same scattering data would allow for another wedge algebra $\mathcal{B}(W) \neq \mathcal{A}(W)$, the vector $\Psi \in H(W)$ is associated with another operator $\Psi=F_{\psi, \mathcal{B}(W)} \Omega$ where $H(W)$ contains all those in- or out- n-particle vectors which are in the domain of $\Delta_{W}^{\frac{1}{2}}$ which form a dense set. We have to show that this cannot occur.

Let us assume that we are dealing with a state-vector of the special form $\Psi=A \Omega, A \in \mathcal{A}(W)$. With respect to the $\mathcal{B}(W)$ algebra there exists a unique affiliated densely defined closed operator $F$ with 9 ]

$$
\begin{aligned}
& A \Omega=F \Omega \\
& F \eta \mathcal{B}(W)
\end{aligned}
$$

where in the last line we used the standard notation $\eta$ for a possibly unbounded closed operator affiliated with $\mathcal{B}(W)$. This forces in particular the inner products with the n-particle out state vectors ${ }^{6}$ to be the same

$$
{ }^{\text {out }}\left\langle p_{n} \ldots p_{1}|A| \Omega\right\rangle={ }^{\text {out }}\left\langle p_{n} \ldots p_{1}|F| \Omega\right\rangle
$$

The physical interpretation would consist in stating that the vectors which $A$ and $F$ generate from the vacuum do not only possess the same particle component (as it would be necessary for obtaining the same asymptotic states with identical normalizations), but their full vacuum polarization clouds (if the operators are charged, these clouds consists of particle/antiparticle pairs.) are identical as well. From this we would like to conclude the equality of their generalized formfactors which then yields the equality of their matrix-elements between particle states with multi-particle ket vectors and hence to the identity $A=F$. For those operators $A \in \mathcal{A}(W)$ which are localized in a double cone $\mathcal{A}(\mathcal{O}) \subset$ $\mathcal{A}(W)$ the LSZ-formalism and on-shell analytic continuation lead to the crossing symmetry (see appendix)

\footnotetext{
${ }^{6}$ Here and in the following we omit the smearing with one-particle wave functions which is necessary to convert improper (plane-wave) vectors into proper ones.
} 


$$
\begin{aligned}
& { }^{\text {out }}\left\langle p_{1}, p_{2}, \ldots p_{l}|A| q_{1}, q_{2} \ldots q_{k}\right\rangle^{\text {in }}= \\
& \underset{p_{c} \rightarrow-p}{a . c .}{ }^{\text {out }}\left\langle p_{1}, p_{2}, \ldots p_{l-1}|A| q_{1}, q_{2} \ldots q_{k},\left(\bar{p}_{c}\right)_{l}\right\rangle^{i n}+c . t= \\
& :^{\text {out }}\left\langle p_{1}, p_{2}, \ldots p_{l-1}|A| q_{1}, q_{2} \ldots q_{k},-\bar{p}_{l}\right\rangle^{\text {in }}+\text { c.t. }
\end{aligned}
$$

The notation is the following. The subscript $c$ indicates that the analytic continuation from momenta $p$ on the positive mass shell to their opposite values $-p$ is done via the $2 \times(n-1)$ dimensional complex mass shell ${ }^{7}$. The bar on top of the $p$ is only a reminder that (as demanded by charge conservation) the crossed momentum - $p$ (the end point of the on-shell analytic continuation) is that of an antiparticle (with identical Poincaré characteristics). In this crossing process there arise a $\delta$-function contraction terms (indicated by c.t.) resulting from a contraction of $p_{l}$ with one of the $q$ multiplied with a lower particle formfactors of $A$. In other words the crossing property consists in naively crossing $p$ 's from outging bras to incoming kets and simultaeously analytically continuing from $p$ to $-p$ and conjugating the charge carried by the crossed particle and results in an identity for the connected part of a formfactor, which in analogy with symmetry identities is often (erroneously) called "crossing symmetry". The crossing of the S-matrix results formally for the special case $A=\mathbf{1}$, but it follows slightly different rules since the unit operator cannot create vacuum polarization but only relates incoming state vectors to outgoing with a possible different number of real particles (on-shell creation/annihilation).

In an appendix the reader will be reminded of the "derivation" of crossing in the LSZ-setting of quantum field theory. The necessary on shell analyticity properties have been derived only in very special cases [10. In the present context we will simply assume the crossing property. Its deeper connection with causality, spectral properties and modular theory are inexorably linked with the existence problem and will be taken up in a separate paper.

Starting from the matrix element (11), the successive application of the crossing property (12) allows to obtain connected matrix elements of $A$ between arbitrary bra-out and ket-in particle states by starting from the special vacuum polarization situation caused by a local operator (associated with a compactly localized spacetime region) applied to the vacuum. From the uniqueness of connected part of the out-in formfactors and the knowledge of the S-matix one then derives the uniqueness of the in-in formfactors. If these formfactors are really the matrix elements of a closed operator, the latter is fixed uniquely.

For free Hermitean fields one can easily that see that the crossing relation is a direct consequence of the KMS property for the wedge algebra $\mathcal{A}(W)$ and its

\footnotetext{
${ }^{7}$ The process of analytic continuation takes place on the complex mass shell and links the forward and backward parts 10 (see appendix); unfortunately the same terminology is often used in case of an analytic connection which passes into the complex off-shell region which can be established much easier.
} 
affiliated smeared fields. We use the following notation

$$
\begin{aligned}
& A(f) \equiv \int A(x) \hat{f}(x) d^{4} x=a^{*}(f)+h . c . \\
& a^{*}(f)=\int a^{*}\left(p\left(\theta, p_{\perp}\right)\right) f\left(p\left(\theta, p_{\perp}\right)\right) \frac{d \theta}{2} d p_{\perp} \\
& A(x)=\frac{1}{(2 \pi)^{\frac{3}{2}}} \int\left(a^{*}\left(p\left(\theta, p_{\perp}\right)\right) e^{i p\left(\theta, p_{\perp}\right) x}+\text { h.c. }\right) \frac{d \theta}{2} d p_{\perp}
\end{aligned}
$$

The $f$ are the wave functions obtained from the W-localized test functions $\hat{f}$, supp $\hat{f} \in W$ by restricting their Fourier transforms to the forward mass-shell

$$
p\left(\theta, p_{\perp}\right)=\left(m_{e f f} \operatorname{ch} \theta, m_{e f f} \operatorname{sh} \theta, p_{\perp}\right), m_{e f f}=\sqrt{m^{2}+p_{\perp}}
$$

In the case of the connected 3-pointfunctions we have

$$
\begin{aligned}
& \left(\Omega, A a^{*}\left(f_{2}\right) a^{*}\left(f_{1}\right) \Omega\right)=\left(\Omega, A A\left(\hat{f}_{2}\right) A\left(\hat{f}_{1}\right) \Omega\right)^{c}, A \in \mathcal{A}(W), A\left(\hat{f}_{i}\right) \eta \mathcal{A}(W) \\
& \stackrel{K M}{=}\left(\Omega, A\left(\hat{f}_{1}\right) \Delta A\left(\hat{f}_{2}\right) \Omega\right)^{c}=\left(\Delta^{\frac{1}{2}} J A\left(\hat{f}_{1}\right) \Omega, A a^{*}\left(f_{2}\right) \Omega\right)^{c} \\
& \curvearrowright \iint\left\langle 0|A| p_{2}, p_{1}\right\rangle f_{2}\left(p\left(\theta_{2}, p_{\perp, 2}\right) f_{1}\left(p\left(\theta_{1}, p_{\perp, 1}\right)\right) d \theta_{2} d p_{\perp, 2} d \theta_{1} d p_{\perp, 1}=\right. \\
& =\iint f_{1}\left(( p ( \theta _ { 1 } + i \pi , - p _ { \perp , 1 } ) ) \langle p _ { 1 } | A | p _ { 2 } \rangle ^ { c } f _ { 2 } \left(p\left(\theta_{2}, p_{\perp, 2}\right) d \theta_{2} d p_{\perp, 2} d \theta_{1} d p_{\perp, 1}\right.\right.
\end{aligned}
$$

By contour shift, the use of analytic properties of matrix elements of W-localized operators in free field theories and the denseness of the W-localized wave function spaces one obtains the desired crossing relation

$$
\left\langle 0|A| p_{2}, p_{1}\right\rangle=\left\langle p\left(\theta_{1}-i \pi,-p_{\perp, 1}\right)|A| p_{2}\right\rangle^{c} \equiv\left\langle-p_{1}|A| p_{2}\right\rangle^{c}
$$

where the right hand side with the backward momentum in the bra vector is a shorthand notation for the analytic continuation in $\theta$. This rather trivial special illustration has an immediate generalization to an arbitrary number of particles with arbitrary spin and internal charges ${ }^{8}$. Hence in the interaction-free case, crossing for formfactors (i.e. bilinear forms of wedge-localized operators, in particular of local composite fields) between multiparticle states follows from the thermal KMS property for wedges (the Unruh situation) and modular oneparticle properties.

The breakdown of this argument in the presence of interactions (in which case crossing has the form (12) ) is related to the fact that generally the multiparticle in and out state vectors cannot be created by operators affiliated to the wedge. There exists however a curious exception for those interacting situations in which wedge-localized so called "tempered PFG" exist. These are operators which are localized in $W$ and whose one-fold application to the vacuum creates

\footnotetext{
${ }^{8}$ In that case the modular $J$ operator involves a charge conjugation and a -1 twist factor
} for halfinteger spin. 
a one-particle state without any vacuum-polarization admixture 9$]^{9}$ (PFGs for sub-wedge localization regions would immediately lead back to free fields) and have reasonable domain properties with respect to translations (the temperateness assumption), . The only known realizations for such situations with Bosons/Fermions are the $\mathrm{d}=1+1$ factorizing models and it is believed that the family of these models exhaust the possibilities of $d=1+1$ tempered PFGs.

General PFGs always exist in any QFT, however the temperateness restriction allows only elastic interactions in $\mathrm{d}=1+1^{10}$ [9. In fact the only known models with real particle number conservation are those where the Fourier transforms of the PFG's $G_{W}(x)$ [5] fulfill a Zamolodchikov-Faddeev [6] algebra which expresses conservation of particles and individual momenta and which in the simplest case of a selfconjugate particle reads

$$
\begin{aligned}
G_{W}(x) & =\frac{1}{\sqrt{2 \pi}} \int\left(e^{-i p x} Z(\theta)+h . c .\right) d \theta, \quad p=m(\operatorname{ch} \theta, \operatorname{sh} \theta) \\
Z(\theta) Z\left(\theta^{\prime}\right) & =S\left(\theta-\theta^{\prime}\right) Z\left(\theta^{\prime}\right) Z(\theta) \\
Z(\theta) Z^{*}\left(\theta^{\prime}\right) & =S^{-1}\left(\theta-\theta^{\prime}\right) Z^{*}\left(\theta^{\prime}\right) Z(\theta)+\delta\left(\theta-\theta^{\prime}\right)
\end{aligned}
$$

The unitarity of the structure functions $S(\theta)$ is a consequence of the *algebra property of the $Z^{\prime} s$ whereas the KMS property of the $G_{W}$-correlation functions with one additional $A \in \mathcal{A}(W)$ follows from the crossing property of the S-matrix; in fact within the setting of factorizing S-matrices the crossing property is equivalent to that KMS property which constitutes the thermal characterizes of wedge localization. The $Z^{\prime} s$ have a simple representation in a bosonic/fermionic Fock space ${ }^{11}$. Each operator $A$ affiliated with $\mathcal{A}(W)$ has a formal power series expansion

$$
A=\sum \frac{1}{n !} \int_{C} \ldots \int_{C} a_{n}\left(\theta_{1}, \ldots \theta_{n}\right): Z\left(\theta_{1}\right) \ldots Z\left(\theta_{n}\right):
$$

where $Z(\theta-i \pi)=Z(\theta)^{*}$, and each integration path $C$ extends over the upper and lower part of the rim of the strip. The strip-analyticity of the coefficient functions $a_{n}$ expresses the wedge-localization of $A$. The sharpening to double cone localization by the intersection of wedges leads to meromorphic functions which obey the kinematical pole condition of Smirnov [7.

Expansions like (14) are nothing more than a generating operator for the formfactors i.e. bilinear forms which fall short of being genuine operators with domains and closures. They are analogous to the LSZ expansions of Heisenberg fields into (asymptotic) free fields. For our above uniqueness argument this is enough, but for a constructive approach this is insufficient.

\footnotetext{
${ }^{9}$ Temperedness restrictions for nonlocal creation anyonic operators actually appeared first in 16$]$.

${ }^{10}$ Tempered PFGs in $\mathrm{d}=1+3$ theories are inconsistent with interactions; in fact there exist arguments that interactions in that case always imply the presence of inelastic scattering [17.

${ }^{11}$ In the presence of one $Z^{\#}$-generator describing several particles (bound states obeying "nuclear democracy") it is simpler to characterize $Z^{\#}$ by its action on state vectors in the multiparticle Fock space rather than by its algebraic commutation structure as in (13).
} 
Although the coefficient functions $S(\theta)$ of the $Z$-algebra turn out to be the 2-particle scattering matrix, there is no need to know this for the calculations: absence of real particle production, wedge-localization and the related KMS property (i.e. spacetime properties) are enough [8].

The case without the temperateness assumption also starts from formfactors between the dense set of wedge affiliated n-particle-ket-states and the bravacuum (which according to modular theory must be equal for the two putative theories).The argument then uses crossing symmetry for the successive movement of particles from the ket to the bra state (and in this way bypasses domain issues).

The asymptotic states in the $\Delta^{\frac{1}{2}}$ domain have a tensor product structure in terms of one-particle states. If their wedge representatives $F_{n} \Omega$ would inherit this factorization structure in the form $F_{n} \Omega=F_{n-1} G \Omega=F_{n-1} \Omega \times G \Omega$ with $G$ being a PFG, then the sequential crossing from bras to kets would follow from the KMS formula of wedge localization. But I have not been able to derive such a factorization from the known domain properties of wedge affiliated $F^{\prime} s$ in the general nontemperate case and I doubt that it holds. This problem is related to the fact that the use of PFG's for the construction of wedge algebras is restricted to factorizing models [9], in realistic interacting theories they do not seem to be useful generators of wedge algebras.

For a recent discussion of how the wedge algebras $\mathcal{A}(W)$ are related to their holographic projections onto the (upper) horizon $\mathcal{A}\left(R_{+}\right)$we refer to [14 15.

\section{Related problems, outlook}

We have seen that by combining modular theory (which among other things gives mathematical precision to the statevector-operator relation) with the crossing property (which permits to elevate relations involving vacuum-polarization formfactors to formfactors describing real particle creation), one obtains a uniqueness argument for the inverse problem in QFT namely a physically admissible S-matrix has, in spite of the myriads of interpolating fields, at most one system of local algebras i.e. at most one field-coordinatization-independent algebraic QFT.

Whereas the crossing property for interaction-free-theories and the closely related $\mathrm{d}=1+1$ dimensional factorizing- models follows from the basic principles of local quantum physics to wedge localization (in fact it is equivalent to the thermal KMS property), the problem of crossing in the general setting remains open. In view of the fact that crossing is the deepest and still mysterious aspect of scattering theory in local quantum physics, its present use as a working hypothesis for the study of the inverse scattering problem is at best preliminary state of affairs.

Implementations of the principles of local quantum physics which try to bypass ultraviolet aspects of the standard approach by placing on-shell objects as formfactors ${ }^{12}$ into the center touch upon age old problems of particle physics,

${ }^{12}$ The S-matrix may be viewed as a formfactor of the identity operator between multi- 
which despite the passage of time have lost nothing of their importance. Beginning as far back as Heisenberg's S-matrix proposal [18, there was the desire to avoid the short distance problems of pointlike field theory by advocating a pure S-matrix theory. Different from the dual model and the subsequent string theory which are also in some sense consequences of this quest, the original intention of the S-matrix approach was to achieve ultraviolet finiteness by maintaining the principles of QFT, but finding concepts which favor the use of on-shell quantities instead of integrating over off-shell vacuum fluctuation of pointlike objects. For a recent review of these old attempts and the many auxiliary concepts and working hypothesis as Mandelstam representations, Regge poles, etc. [19] 20].

The present modular setting subjects this old approach to a critical review. Although there is agreement with its basic premise that on-shell concepts should play an important role right from the beginning and that there should exist a different way to introduce interactions than by coupling free fields, the new approach would not abandon the causality and localization principles of QFT as was advocated in the old approach. The message would rather be that one should avoid the use of pointlike fields (and their time-ordered correlation functions) in intermediate steps of the calculation. Once the net of spacetimeindexed operator algebras has been constructed by other means, there is no harm to use these singular objects (operator-valued distributions) as generators of these operator algebras and present the operator-algebra content in terms of field coordinatizations.

There are some fine points in such a program which need to be taken into account. A useful representative illustration is provided by the interaction-free theories which correspond to the zero mass "continuous spin" (spin-tower) representations which appear in Wigner's classification of irreducible Poincaré-group representations. It is a characteristic property of this representation that the compactly modular localized subspaces are empty and the best localized (smallest localization region) nontrivial subspaces have a semiinfinite string (instead of a point) as the core of their localization [21] 22]. In this case the associated operator algebras (which are obtained by applying the Weyl functor to the oneparticle localization-subspaces) admit only string-like generators. One expects that this net of algebras possesses a net of pointlike localizable subalgebras of observables which consist of string/anti-string such that by the use of the Doplicher-Roberts reconstruction the original string-localized net appears as a "DR field-algebra" 3. These investigations suggest that the positive energy restriction guaranties that one never needs to work with generators which have worse than spacelike cone localization region (with semiinfinite strings as their core). In the presence of a mass gap this can be rigorously shown 24. For conformally invariant theories the result in [23] strongly suggest that pointlike generators are always available.

The bootstrap formfactor approach to factorizing models may serve as an excellent illustration of this new ultraviolet-finite way of thinking about QFT

particle in and out vectors. The mixed (out-in) formfactors may be converted into (in,in) formfactors i.e. to sequilinear forms of (singular) operators with repect to the basis of invectors. 
(see 31] for a review within the present setting of scattering theory). Here one bypasses the technical frontiers between renormalizable/nonrenormalizable interactions by using a different approach to Lagrangian quantization or causal perturbation of free fields. For $\mathrm{d}=1+1$ factorizing models this is achieved by starting with an algebraic structure which avoids pointlike fields in favor of operators fulfilling a Z-F algebra consistent with wedge-like localization. With other words, the system of wedge algebra $\mathcal{A}(W)$ is constructed before any pointlike field appears on the scene. The next step, namely to get from noncompact wedges to compact localization regions, consists in the formation of double cone intersection algebras; this leads to the so-called kinematical pole equation which relates the lower with the higher formfactors and defines the formfactor spaces for double-cone localized objects. Whereas the Z-F algebra generators are PFGs, the double-cone localization leads to the vacuum polarization clouds in form of expansions with respect to Z-F operators which resemble the LSZ expansions of Heisenberg operators in terms of incoming free fields (14). The finite size of the spacetime extension of the double-cone localized [5] operators shows up in form of a Payley-Wiener asymptotic behavior of the meromorphic formfactors.

The use of pointlike fields within formfactors (i.e. avoiding correlations) causes no problems; the pointlike nature is reflected in a polynomial behavior in certain reduced formfactors 31. The point which needs to be emphasized here is there is no ultraviolet limitation coming from power-counting and leading to the standard separation into renormalizable and nonrenormalizable coupling; every admissable factorizing S-Matrix leads to power bounded formfactors in terms of a few physical parameters which were already present in the S-matrix. Presently it appears that any S-matrix with the crossing property and one-particle poles (obtained by applying the fusion rules to the given 2-particle S-matrix) leads to the formfactors of an existing QFT. Any potential further restriction on the pole structure must come from the modular wedge localization requirement. One needs investigations which go beyond 8$]$ in order to clarify this point.

It is comforting to observe that the limitation of Lagrangian fields (which must have operator dimension near the canonical free field dimension in order to maintain renormalizability) disappears in this bootstrap-formfactor setting; in fact any factorizing S-matrix always leads to a renormalizable theory in the sense of polynomial bounded high energy behavior and a finite number of physical parameters). So the standard renormalizable/nonrenormalizable separation according to short distance behavior becomes void; short distance properties (of what? there is no preferred Lagrangian field coordinate!) are simply not part of the modular program and "nonrenormalizable" in the Lagrangian sense presumably corresponds to the triviality of algebras localized in double cone intersections in the present setting. The remaining question about existence is whether the double cone intersection algebras are nontrivial in the sense of formfactors and whether these formfactors are really coming from operators (or whether the correlation functions exist and have the right properties).

The crucial question is whether a construction based on formfactors and modular concepts is also conceivable with realistic S-matrices which describe on- 
shell particle creation approach. The present uniqueness argument of the inverse problem suggests that this should indeed be expected. The fact that the crossing property is an equation for the connected part of the formfactors and not for the matrix elements themselves complicates its encoding into an operator approach. There are arguments in favor of existence of an auxiliary operator formalism for connected formfactors of very special objects. These attempts at a general on-shell construction require new concepts and will be presented in a separate paper [25]. As the standard perturbation theory, the formfactor approach should admit iterative solution with the tree approximation as an input. Since such an approach does not require to deal with correlations between several pointlike fields (i.e. with singular short distance fluctuations), short distance problems and the related ultraviolet divergencies do not enter. Naturally one expects that the S-matrix and formfactors of renormalizable theories in the standard sense to be also solutions in the new setting.

In particular one expects a clarification of the ghost issue. This is because the necessity to introduce cohomological BRST ghosts is inexorably related with controlling the short distance fluctuations in intermediated steps of the Feynman approach. But since they leave no traces in the physical operator algebras after the cohomological descend to the physical space (similar to catalyzers in chemistry [32]), there is no place for ghosts in an approach which bypasses the short distance aspects.

In this way one may hope for a return of Heisenberg's credo that quantum physics should admit a formulation solely in terms of observables. In fact local quantum physics provides an excellent illustration of the power of this idea. The locality structure is such a strong restriction that the structure of the (neutral) observable subalgebras allows to reconstruct the statistics (spacelike commutation structure) and the internal symmetry of the charge-carrying field algebra from its observable "shadow" 3. This is a perfect local analog of Marc Kac's aphorism with which we started this paper. The uniqueness and constructive existence of the inverse scattering problem extends this analogy to the global (asymptotic) domain. In quantum mechanics this is only possible under severe restrictions on the interaction potential.

New ideas for which one has only very special illustrations are sometimes easier communicated by pointing to the underlying philosophy. It has been known that without the presence of interaction terms, i.e. for free Wigner particles with arbitrary spin/helicity, there does indeed exist a modular approach leading directly to the net of algebras. In this case the S-matrix input trivializes to the Wigner one-particle representation space and the wedge localization amounts to a construction of geometrically defined real subspaces and their intersections [4 [21]. The application of the Weyl- (or CAR-) functor to these subspaces yields a system of extended generators of the net of von Neumann algebras.

The standard method which uses pointlike fields is different, although it also starts from the same Wigner one-particle representations. Pointlike covariant fields result from intertwiners between the unique $(\mathrm{m}, \mathrm{s})$ Wigner representations and the multitude of so-called covariant representations 34 which form a infinite 
denumerable set. In this way one obtains infinitely many covariant fields which live in the same multiparticle tensor Fock space and which define different field coordinatizations of the same algebraic net, i.e. the uniqueness of the Wigner $(\mathrm{m}, \mathrm{s})$ representation theory gets lost in the construction of the fields and is recovered by passing to the net of algebras. Actually this system of fields from different choices of intertwiners exhausts only the linear part of the system of all possible pointlike field coordinatizations. The full system is identical to the Wick polynomials formed from the linear system. The analog system in the case with interactions is the Borchers class of the theory ${ }^{13}$. The interacting case also starts with Wigner particle data, this time one needs in addition an invarant quantitative characterization of their interaction which is the S-matrix. The latter characterizes the position of the dense subspace of wedge-localized state vectors within the Fock space of incoming particles. The remaining problem of how to go from here to the net of subalgebras is the difficult step which needs new conceptual and mathematical constructs.

I believe that it is fruitful to view the general modular approach as an extension of Wigner's program. Wigner was the first who succeeded to describe particles in a completely intrinsic manner without using "quantization" of classical fields. Therefore the program advocated in this paper should be viewed as a generalization of Wigner's approach to full QFT in the presence of interactions.

\subsection{Appendix: Crossing symmetry with LSZ reduction}

Crossing has been first observed in Feynman perturbation before a formal derivation was given in the setting of LSZ scattering theory. Its formal aspects are easily obtained from the LSZ asymptotic convergence

$$
\begin{gathered}
\lim _{t \rightarrow \mp \infty} A^{\#}\left(f_{t}\right) \Phi=A^{\#}(f)_{\text {in }, \text { out }} \Phi, A^{\#}=A \text { or } A^{*} \\
A\left(f_{t}\right)=\int f_{t}(x) U(x) A U^{*}(x) d^{4} x, A \in \mathcal{A}(\mathcal{O}) \\
f_{t}(x)=\frac{1}{(2 \pi)^{2}} \int e^{i\left(p_{0}-\omega(p)\right) t-i p x} f(\vec{p}) d^{4} x, \omega(p)=\sqrt{\vec{p}^{2}+m^{2}}
\end{gathered}
$$

which can be derived on a dense set of states. This is known to lead to the wellknown reduction formulas 29] 30] (leaving out the smearing with one-particle wavefunctions) which in terms of connected matrix elements read

$$
\begin{aligned}
& \left.{ }^{\text {out }}\left\langle q_{1}, q_{2}, \ldots q_{m}|F| p_{1}, p_{2} \ldots p_{n}\right\rangle^{i n}\right|_{\text {conn }}= \\
& -i \int{ }^{\text {out }}\left\langle q_{2}, \ldots q_{m}\left|K_{y} T F A^{*}(y)\right| p_{1}, p_{2} \ldots p_{n}\right\rangle^{\text {in }} d^{4} y e^{-i q_{1} y}= \\
& -i \int{ }^{\text {out }}\left\langle q_{1}, q_{2}, \ldots q_{m}\left|K_{y} T F A(y)\right| p_{2} \ldots p_{n}\right\rangle^{i n} d^{4} y e^{i p_{1} y}=
\end{aligned}
$$

\footnotetext{
${ }^{13}$ One obtains a very interesting generalization of this situation for so-called generalized free fields 33 . An analysis of that situation in the present setting of modular subspaces and Weyl functors could lead to simplifications and new insights.
} 
Here the time-ordering $T$ between the original operator $F \in \mathcal{A}(\mathcal{O})$ and the interpolating Heisenberg field $A(x)$ or $A^{*}(x)$ appears in the reduction of a particle from the bra- or ket state. For the definition of the time ordering of a fixed finitely localized operator $F$ and a field with variable localization $y$ we may use $T F A(y)=\theta(-y) F A(y)+\theta(y) A(y) F$, however as we place the momenta on-shell, the definition of time ordering for $y$ near $\operatorname{loc} F$ is irrelevant ${ }^{14}$. Each such reduction is accompanied by another disconnected contribution in which the creation operator of an outgoing particle say $a_{\text {out }}^{*}\left(q_{1}\right)$ changes to an incoming $a_{i n}\left(q_{1}\right)$ acting on the incoming configuration (and the opposite situation i.e. $\left.a_{\text {in }}^{*}\left(p_{1}\right) \rightarrow a_{\text {out }}\left(p_{1}\right)\right)$. These terms (which contain formfactors with one particle less in the bra- and ket- vektors) have been omitted since they do not contribute to generic nonoverlapping momentum contributions and to the analytic continuations. Under the assumption that there is an analytic path from $p \rightarrow-p$ (or $\theta \rightarrow \theta-i \pi$ in the wedge adapted rapidity parametrization) the comparison between the two expressions gives the desired crossing symmetry: a particle of momentum $\mathrm{p}$ in the ket state within the connected part of a formfactor is indistinguishable from a bra antiparticle at momentum -p (here denoted as $-\bar{p})$.

In order to obtain that required analytic path on the complex mass-shell of the $2 \rightarrow 2$ scattering amplitude it is convenient to pass from time ordering to retardation

$$
T F A(y)=R F A(y)+\{F, A(y)\}
$$

The unordered (anticommutator) term does not have the pole structure on which the Klein-Gordon operator $K_{y}$ can have a nontrivial on-shell action and therefore drops out. The application of the JLD spectral representation puts the p-dependence into the denominator of the integrand of an integral representation where the construction of the analytic path proceeds in a completely analog fashion to the derivation of crossing for the S-matrix [35] [30. Whereas it is fairly easy to find an off-shell analytic path, the construction of an on-shell path which remains in the complex mass shell is a significantly more difficult matter [10.

The simplifications of the LSZ formalism resulting from factorizability of models can be found in an appendix of 31.

\section{References}

[1] S. Doplicher and J. E. Roberts, Commun. Math. Phys. 131, (1990) 51

[2] A. S. Wightman and R. F. Streater, Spin, Statistics and all That, Benjamin Inc. New York 1964

[3] R. Haag, Local Quantum Physics, Springer 1992

[4] B. Schroer, Annals of Physics Vol. 255, No.2, 270 (1997)

\footnotetext{
${ }^{14}$ These on-shell reduction formulas remain valid if one used as interpolating pointlike fields $A(x)$ the translates of bounded localized operators [30.
} 
[5] B. Schroer, J. Math. Phys. 41, (2000) 380

[6] A. B. Zamolodchikov and AL. B. Zamolodchikov, Ann. Phys. 120, (1979) 253, L. D. Faddeev, Sov. Sci. Rev. Math. Phys. C1, (1980) 107

[7] F. A. Smirnov, Int.J. Mod. Phys. A4, (1989) 156. Our operator notation is more close to M. Yu Lashkevich, Sectors of Mutually Local Fields in Integrable Models of Quantum Field Theory, hep-th/9406118 unpublished

[8] G. Lechner, Polarization-Free Quantum Fields and Interactions, hep-th/0303062

[9] H.J. Borchers, D. Buchholz and B. Schroer, Commun. Math. Phys. 219, (2001) 125, hep-th/0003243

[10] J. Bros, H. Epstein and V. Glaser, A Proof of the Crossing Property for Two-Particle Amplitudes in General Quantum Field Theory, Commun. Math. Phys. 1, (1965) 240

[11] D. Buchholz and K. Fredenhagen, Commun. Math. Phys. 56, (1977) 91

[12] H.-J. Borchers. JMP 41 (2000) 3604

[13] A. Capelli, C. Itzykson and J. B. Zuber, CMP 113, (1987) 1

[14] B. Schroer, JPA 35, (2002) 9165. B. Schroer, IJMPA 18, (2003) 1671 (hep-th/0208113)

[15] L. Fassarella and B. Schroer, Phys. Lett. B 538, (2002) 415

[16] J. Mund, Lett. Math. Phys. 43, (1998) 319.

[17] S. Åks, Journ. Math. Phys. 6, (1965) 516

[18] W. Heisenberg, Zeit. für Naturforschung, 1 (1946) 608

[19] R. J. Eden, P. V. Landshoff, D. I. Olive and J. C. Polkinghorne, The analytic S-Matrix, Cambridge Univ. Press, Cambridge 1966

[20] A. R. White, The Past and Future of S-matrix Theory, hep-th/0002303

[21] R. Brunetti, D. Guido and R. Longo, Rev.Math.Phys. 14, (2002) 759

[22] L. Fassarella and B. Schroer, J. Phys. A: Math. Gen.35, (2002) 9123

[23] M. Jörss, Lett. Math.Phys. 38, (1996) 257

[24] D. Buchholz and K. Fredenhagen, Locality and the structure of particle states, Math. Phys. 84, (1982) 1

[25] B. Schroer, An operator implementation of crossing based on the "Masterfield" hypothesis, in preparation 
[26] H.-J. Borchers and J. Yngvason, On the PCT Theorem in the Theory of Local Observables, math-ph/0012020

[27] J. Mund, Ann. H. Poinc. 2, (2002) 907

[28] A. Connes, Ann. Inst. Fourier 24, (1974) 121

[29] K. Hepp, On the connection between Wightman and LSZ quantum field theory, page 135 in: Brandeis University Summer Institute in Theoretical Physics 1965, "Axiomatic Field Theory" Volume 1, ed. (M. Chretien and S. Deser ed.), Gordon and Breach 1966

[30] H. Araki, Mathematical Theory of Quantum Fields, Int. Series of Monographs in Physics, Oxford University Press 1999

[31] H. Babujian, A. Fring, M. Karowski and A. Zapletal, Nucl. Phys. B 538, (1999) 535, H. Babujian and M. Karowski, Exact formfactors in integrable quantum field theories, the sine-Gordon model (II), hep-th/0105178

[32] M. Duetsch and B. Schroer, J. Phys. A: Math. Gen. 33, (2000) 4317

[33] M. Duetsch abd K.-H. Rehren, Generalized free fields and the AdS-CFT correspondence, math-ph/0209035

[34] S. Weinberg, The Quantum Theory of Fields I, Cambridge University Press 1995

[35] N. N. Bogoliubov, A. A. Logunov, A. I. Oksak and I. T. Todorov, General Principles of Quantum Field Theory, Kluwer Academic Publishers 1990 